\title{
A Rare Case of Incidentally Diagnosed Brucellosis in a Patient with Congenital Dyserythropoietic Anemia and IgA Nephropathy: A Diagnostic Dilemma
}

\author{
Kundu Ramit ${ }^{1}$, Priyadarshi Ketan ${ }^{1}$, Veerappan Kowsalya ${ }^{1}$, B. S. Nagashri ${ }^{2}$, \\ Surendran Deepanjali ${ }^{2}$ and Sastry Apurba Sankar ${ }^{1 *}$
}

${ }^{1}$ Department of Microbiology, ${ }^{2}$ Department of Medicine, Jawaharlal Institute of Postgraduate Medical Education and Research (JIPMER), Puducherry - 605006, India

*Corresponding author

\begin{abstract}
A B S T R A C T
Keywords

Brucellosis, IgA nephropathy, Automated blood culture

Article Info

Accepted:

22 April 2021

Available Online:

10 May 2021

Brucellosis continues to be a significant yet frequently neglected threat to both human and veterinary health. However, the disease remains a diagnostic challenge for most healthcare settings due to the non-specific clinical findings associated with the disease and relative inexperience of both clinicians and laboratories in the identification of the disease as well as its causative agent. We report a case of laboratory-confirmed brucellosis from a tertiary-care hospital in southern India, diagnosed incidentally in a 20-year-old male presenting with acute onset fever along with multisystem signs and symptoms who was previously diagnosed with congenital dyserythropoietic anemia and IgA Nephropathy.
\end{abstract}

\section{Introduction}

Human brucellosis, also known under the names of - Bang's disease, Malta fever, undulant fever, and Mediterranean fever, is an important re-emerging zoonosis with a worldwide distribution. ${ }^{(1)}$

Accurate diagnosis of human brucellosis is difficult, due to non-specific clinical presentations. ${ }^{(2)}$ Laboratory identification in resource-limited settings poses a significant problem, as the non-availability of automated blood culture systems and automated identification systems makes the diagnosis delayed and inaccurate.

We describe a challenging case of human brucellosis diagnosed incidentally on blood culture in a 20-year-old male patient previously diagnosed with congenital dyserythropoietic anemia type II and $\operatorname{Ig} \mathrm{A}$ nephropathy, who presented with signs and symptoms pertaining to multiple organ systems. 


\section{Case Presentation}

A 20-year-old male from southern India presented to our hospital with chief complaints of moderate to high-grade fever, intermittent in nature, not associated with chills / rigor / night sweats for the past 3 days. He also complained of persistent pain and distension of the abdomen for the last two weeks along with few episodes of vomiting which was nonbilious, non-projectile, and not bloodstained. He also had complaints of sore throat and cough with mild mucoid expectoration along with nasal stuffiness. He also reported bilateral knee and ankle joint pain of moderate intensity with mild lower leg swelling, along with occasional burning sensation during micturition. He had no history of difficulty in breathing, headache, or neck stiffness and had normal urine output. In the past, he was diagnosed with - (1) Congenital dyserythropoietic anemia type II ten years back (2) IgA Nephropathy nine years ago, for which he received treatment with azathioprine and steroids for 3 years (3) Immune complex glomerulonephritis - possibly post-infectious diagnosed two years back on renal biopsy for which he was treated using steroids for two months. On presentation, the patient was febrile with moderate pallor and mild icterus. Mild splenomegaly, but no lymphadenopathy or pedal edema were noted. The abdomen was soft and mildly distended with tenderness over the bilateral loin and flank region and no shifting dullness. He had bilateral tonsillar enlargement with posterior pharyngeal wall congestion.

On admission, urine and paired blood culture samples were sent before starting on intravenous cefoperazone-sulbactam and azithromycin empirically. Hemogram showed leukocytosis (Total Leukocyte Count of 19900 $/ \mu \mathrm{L}$ ) with anemia (hemoglobin $8 \mathrm{~g} / \mathrm{dL}$ ). Chest $\mathrm{X}$-ray was normal. In view of the upper respiratory tract symptoms, SARS-CoV2rRT-
PCR test was performed which turned out negative. Urine culture was sterile at 48 hours. Automated blood culture was performed in VirtuoBacT/ALERT system, which did not flag positive till 48 hours of incubation. Eventually, the blood cultures were flagged positive with a time to positivity (TTP) of 63 hours. Gram staining performed from BacT/ALERT bottle broth showed no microorganisms. Subculture from the broth was performed on 5\% sheep blood agar, chocolate agar, and MacConkey agar. Faintly visible colonies were seen only after 20 - 22 hours of aerobic incubation. The organism isolated was a gram-negative coccobacillus (approx. $1.5 \mu \mathrm{M}$ $\times 1 \mu \mathrm{M}$ ) on gram staining from colony (Figure 1) along with being catalase and oxidase test positive. Identification was performed using Matrix-Assisted Laser Desorption/Ionization Time of Flight Mass Spectrometry (VITEKMS) and identified as Brucella species. Additional tests were performed from the colony growth. Brucella differential stain or Stamp's modification of Ziehl - Neelsen staining ${ }^{(3)}$ was performed in which organism remained reddish-pink coccobacilli (Figure 2). Urea is rapidly hydrolyzed within 20 minutes of incubation. Growth was enhanced on incubation in capnophilic $\left(5-10 \% \mathrm{CO}_{2}\right)$ incubation (Figures 3 and 4). Accurate nomen species-level identification could not be made. Upon identification of Brucella spp. on blood culture, Standard agglutination test (SAT) was also performed on the patient's serum to detect the presence of agglutinating antibodies against Brucella which turned out positive. ${ }^{(4)}$

Following laboratory identification, extensive efforts to elucidate history suggestive of a source of exposure to this zoonotic pathogen were unsuccessful - as even though the patient hails from a rural background, he did not give any history of contact with cattle or other farm animals, does not have any pets at home and there is also no history of consumption of unpasteurized milk or milk products. After the 
laboratory diagnosis of Brucellosis, the patient was started on a specific antimicrobial treatment regimen consisting of - oral rifampicin $600 \mathrm{mg}$ (once daily) and doxycycline $100 \mathrm{mg}$ (every 12 hours) and planned to continue for a total of 6 weeks on discharge. However, intravenous cefoperazone-sulbactam and azithromycin were continued for a total period of 7 days. During his stay in the hospital, the patient experienced symptomatic improvement starting a few days following the institution of antimicrobial therapy (subsiding of fever and improvement of gastrointestinal symptoms). Apart from this, there was also an improvement in the patient's laboratory findings at follow-up (return of total leukocyte count to baseline levels and no growth of Brucella seen in 2 paired blood culture samples sent 5 days after starting antibiotic therapy). The patient continued to receive treatment for pre-existing conditions i.e., IgA Nephropathy and congenital dyserythropoietic anemia type II.

\section{Results and Discussion}

Brucellosis is a neglected zoonosis of public health concern. Human infections due to Brucella species are grossly under-diagnosed and under-reported in South India. There is also a significant possibility for misdiagnosis as the clinical spectrum of the disease can largely overlap with many other conditions (e.g., tuberculosis). ${ }^{(2)}$ However, accurate diagnosis is of utmost importance from a treatment point of view because the recommended regimen is different and specific with rifampicin and doxycycline. Regular antimicrobials with gram-negative coverage are ineffective.

Complexity in the accurate diagnosis of Brucellosis poses a challenge at multiple levels. At a clinical level, the patient often presents with non-specific symptoms and signs or with pyrexia of unknown origin (PUO). In absence of significant occupational or zoonotic contact history, it is challenging to keep brucellosis in the differential diagnosis especially in areas where it is uncommon or less frequently reported.

At the laboratory level, diagnosis of brucellosis is associated with additional difficulties. Laboratory diagnosis mostly revolves around the recovery of viable organisms from the blood by various blood culture techniques (classical broth culture or automated systems like BacT/Alert), however, in most cases the yield of organisms from blood is poor (due to the fastidious, facultative intracellular nature of the organism - requiring prolonged incubation often supplemented with $5-10 \%$ carbon dioxide). ${ }^{(5)}$ Conventional blood cultures are inconsistent in the isolation of Brucella. Automated blood culture systems are capable, but the time to positivity is often high - beyond 48 to 60hours of incubation causing further delay in management. Adequate blood volume during blood culture is a significant limiting factor. Even, from positive flagged bottles, isolation on culture media takes time from 24 to 48 hours or longer sometimes. Identification using conventional methods is very difficult and inaccurate and is usually a good guess with clinical correlation. Automated identification systems (e.g., MALDI-TOF, VITEK-2) are rapid but availability at all places is questionable.

These systems also sometimes give misidentification. So, culture identification of Brucellosis is time taking with a turnaround time of approx. 96 hours on average. Serodiagnostic tests include Standard Tube Agglutination Test (SAT), Rose Bengal Card Test, indirect Coombs' test along with recent modalities such as ELISA and immunocapture agglutination. 
Fig.1 Gram-stained smear from positively flagged blood culture bottle, under 100X magnification (showing gram-negative coccobacilli)

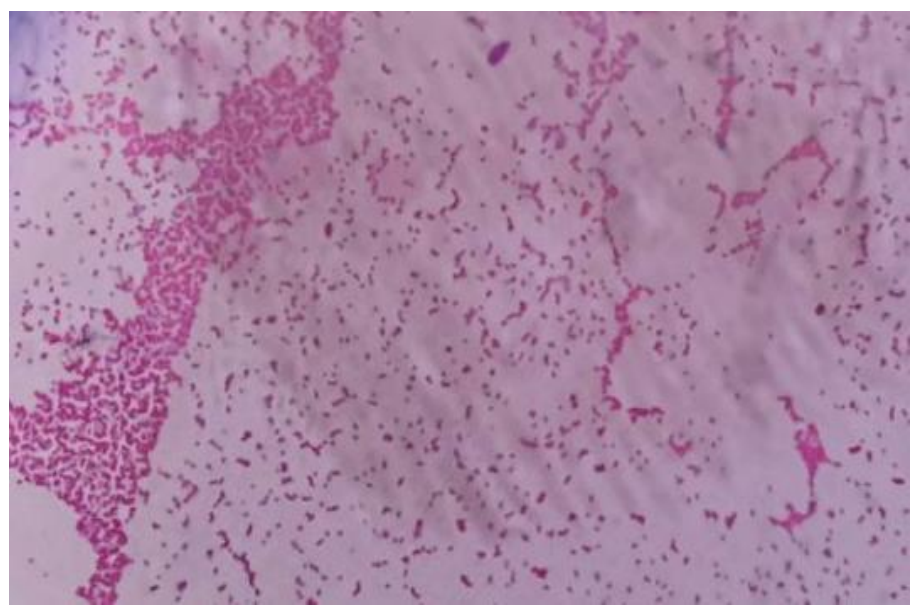

Fig.2 Smear from positively flagged blood culture bottle using Brucella differential stain/Stamp's modification of Ziehl-Neelsen staining, under 100X magnification (showing reddish-pinkacid fast coccobacilli)

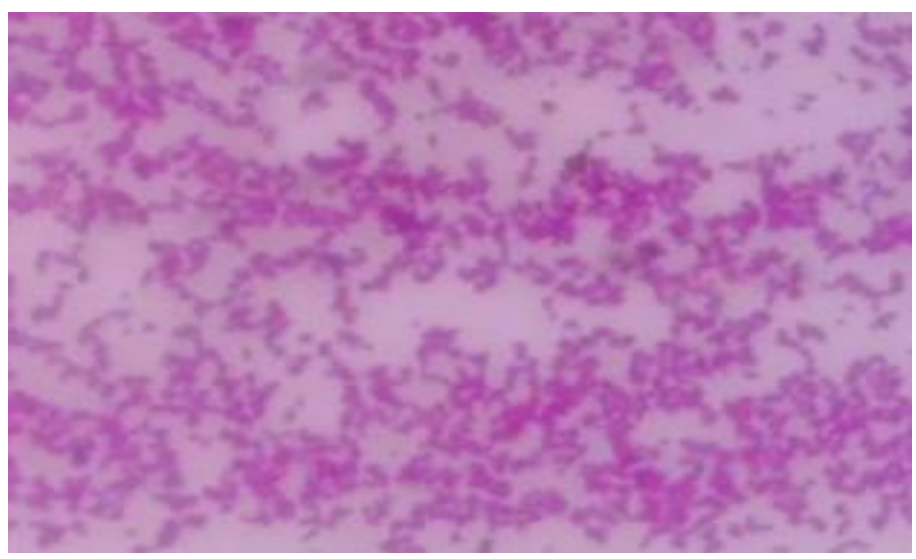

Fig.3 Enhanced growth of Brucella spp. on 5\% sheep blood agar upon capnophilic incubation $\left(5-10 \% \mathrm{CO}_{2}\right)$ at $37^{\circ} \mathrm{C}$

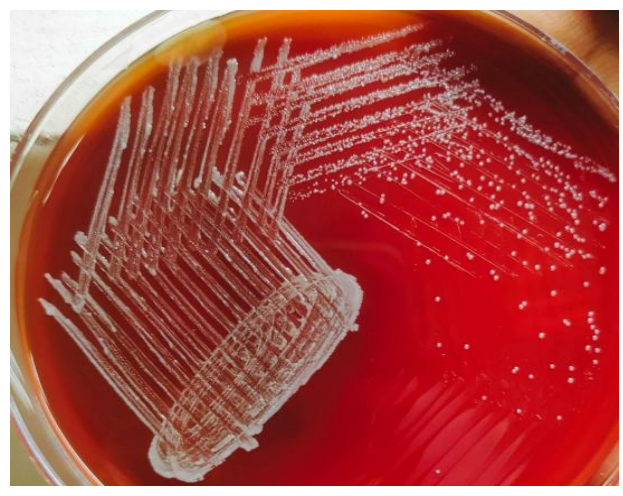


Fig.4 Enhanced growth of Brucella spp. on chocolate agar upon capnophilic incubation $\left(5-10 \% \mathrm{CO}_{2}\right)$ at $37^{\circ} \mathrm{C}$

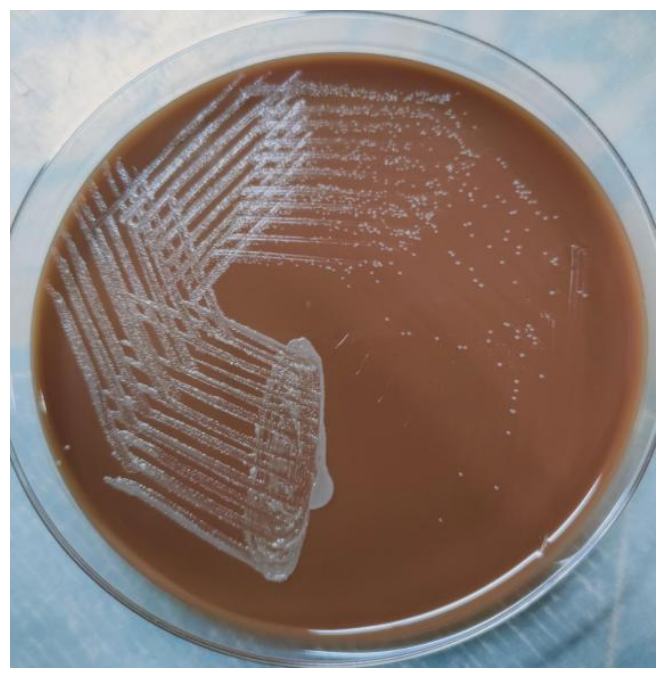

Standard Agglutination tests and ELISA platforms are accurate and rapid, but lack of clinical suspicion limits its usability. ${ }^{(4)}$

Species-level identification generally requires either conventional or multiplex Polymerase Chain Reaction (PCR) performed on various genetic targets such as Int-hyp, Brucellasuis Transposase, and $g l k$. Subtyping of biovars involves molecular techniques such as pulsedfield gel electrophoresis, Single Nucleotide Polymorphism (SNP) analysis, and Multilocus Variable-Number Tandem Repeat (VNTR) Analysis (MLVA). ${ }^{(6)}$ Unfortunately, none of these methods are in routine use even in tertiary care settings in regions where the disease is endemic.

Laboratory identification is generally corroborated with risk factors for exposure obtained from patient history. However, a definitive history of exposure (to livestock, unpasteurized dairy products, etc.) cannot be made out in many cases and the identification of the organism in many of these situations is purely incidental - mostly from blood cultures sent in view of unexplained fever or other non-specific manifestations of disseminated infection - sometimes in patients under treatment for apparently unrelated conditions. ${ }^{(1)}$

In the present case, the patient presented with multisystem signs and symptoms. Clinical diagnosis was a challenge in this patient as the patient had signs and symptoms pertaining to various organ systems of the body - (1) Systemic - e.g., fever (2) Gastro-intestinal e.g., abdominal pain and distension, vomiting (3) Respiratory - e.g., sore throat, cough (4) Musculoskeletal - e.g., joint pain (5) Genitourinary - e.g., dysuria. Pre-existing conditions like Congenital dyserythropoietic anemia type II and IgA Nephropathy also complicated the clinical suspicion of Brucellosis. There was no history suggestive of exposure to this zoonotic pathogen with neither history of contact with cattle or other farm animals or pets at home, nor consumption of unpasteurized milk or milk products. The diagnosis was reached in reasonable time and specific therapy was timely instituted leading to a positive outcome in this case.

A literature review was performed on PubMed using the search term 'Brucella', 'glomerulonephritis', 'IgA nephropathy', and 'Congenital dyserythropoietic anemia'. There are four case reports showing the association of brucellosis with $\operatorname{IgA}$ nephropathy, ${ }^{(7-9)}$ and 
18 published articles of association between brucellosis and glomerulonephritis. ${ }^{(\mathbf{1 0})}$ There was no published literature on brucellosis in cases of Congenital dyserythropoietic anemia.

The diagnosis of human brucellosis is challenging in the absence of typical clinical presentation and history of zoonotic or foodborne exposure. Pre-existing disease conditions may lead to diagnostic confusion. In these cases, blood culture is a valuable modality to diagnose brucellosis. Automated blood culture and identification systems supplemented with serological assays aids in accurate and timely diagnosis which can lead to the early institution of specific antimicrobial therapy and improved patient outcome. Through this case report, we would like to emphasize the deceptive clinical nature of brucellosis and the difficulties associated with arriving at a prompt laboratory diagnosis even in a tertiary health care setting.

\section{References}

1. Al Dahouk S, Tomaso H, Nöckler K, Neubauer H, Frangoulidis D. Laboratorybased diagnosis of brucellosis--a review of the literature. Part II: serological tests for brucellosis. Clinical laboratory. 2003 Jan 1;49(11-12):577-89.

2. Dasari S, Naha K, Prabhu M. Brucellosis and tuberculosis: clinical overlap and pitfalls. Asian Pacific journal of tropical medicine. 2013 Oct 1;6(10):823-5.

3. Joshi P A, Kulkarni R D, Powar R M. Modified cold ZN staining for presumptive identification of Brucella.
Indian J Med Res. 2005 Feb 1;121(2):108-.

4. Gómez M C, Nieto J A, Rosa C, Geijo P, Escribano MA, Munoz A, López C. Evaluation of seven tests for diagnosis of human brucellosis in an area where the disease is endemic. Clinical and Vaccine Immunology. 2008 Jun 1;15(6):1031-3.

5. Yagupsky P. Detection of Brucellae in blood cultures. Journal of clinical microbiology. 1999 Nov 1;37(11):343742.

6. Winchell J M, Wolff B J, Tiller R, Bowen M D, Hoffmaster A R. Rapid identification and discrimination of Brucella isolates by use of real-time PCR and high-resolution melt analysis. Journal of clinical microbiology. 2010 Mar 1;48(3):697-702.

7. Siegelmann N, Abraham A S, Rudensky $\mathrm{B}$, Shemesh O. Brucellosis with nephrotic syndrome, nephritis and IgA nephropathy. Postgraduate medical journal. 1992 Oct 1;68(804):834-6.

8. Parlak E. A case of glomerulonephritis caused by brucellosis. Tropical Doctor. 2020 Oct;50(4):360-1.

9. Nunan $\mathrm{T}$ O, Eykyn $\mathrm{S} J$, Jones $\mathrm{N} F$. Brucellosis with mesangial IgA nephropathy: successful treatment with doxycycline and rifampicin. British medical journal (Clinical research ed.). 1984 Jun 16;288(6433): 1802.

10. Elzouki A Y, Akthar M, Mirza K. Brucella endocarditis associated with glomerulonephritis and renal vasculitis. Pediatric Nephrology. 1996 Nov;10(6):748-51.

\section{How to cite this article:}

Kundu Ramit, Priyadarshi Ketan, Veerappan Kowsalya, B. S. Nagashri, Surendran Deepanjali and Sastry Apurba Sankar. 2021. A Rare Case of Incidentally Diagnosed Brucellosis in a Patient with Congenital Dyserythropoietic Anemia and IgA Nephropathy: A Diagnostic Dilemma. Int.J.Curr.Microbiol.App.Sci. 10(05): 753-758. doi: https://doi.org/10.20546/ijcmas.2021.1005.085 